\title{
La représentation du chamanisme coréen chez l'écrivain Kim Dong-ri (1913-1995)
}

The Literary Representation of Korean Shamanism by Kim Dong-ri (1913-1995)

\section{Min Sook LE}

\section{(2) OpenEdition}

\section{Journals}

Édition électronique

URL : http://journals.openedition.org/transtexts/417

DOI : $10.4000 /$ transtexts.417

ISSN : 2105-2549

Éditeur

Gregory B. Lee

Édition imprimée

Date de publication : 7 avril 2011

ISSN : 1771-2084

Référence électronique

Min Sook LE, «La représentation du chamanisme coréen chez l'écrivain Kim Dong-ri (1913-1995) », Transtext(e)s Transcultures 跨文本跨文化 [En ligne], 6 | 2011, mis en ligne le 07 avril 2011, consulté le 10 décembre 2020. URL : http://journals.openedition.org/transtexts/417 ; DOI : https://doi.org/ $10.4000 /$ transtexts.417 


\section{Transtext(e)s Transcultures 跨文本跨 文化}

Journal of Global Cultural Studies

6 | 2009:

Debating China

\section{La représentation du chamanisme coréen chez l'écrivain Kim Dong-ri (1913-1995)}

The Literary Representation of Korean Shamanism by Kim Dong-ri (1913-1995)

\section{MIN SoOK LE}

\section{Résumés}

Le chamanisme a exercé une influence majeure sur Kim Dong-ri (1913-1995), figure emblématique de la littérature coréenne du XXe siècle. Cette religion autochtone avait connu ses plus beaux jours au royaume de Silla (57 av. J.-C. - 935) avant l'introduction du bouddhisme et du confucianisme qui l'ont supplantée au cours de l'histoire coréenne. Originaire de Gyeongju, ancienne capitale de Silla, Kim essaie de retrouver dans le chamanisme l'âme fondatrice de sa nation et ne cesse de le sublimer dans son œuvre.

Cette étude a pour objet d'élucider les questions suivantes : Comment le chamanisme est-il représenté dans la littérature de Kim ? Par quels moyens valorise-t-il ce fait religieux ? Quel est, enfin, le rapport entre le chamanisme et ce qu'il appelle « troisième humanisme " ou " humanisme coréen " ? L'objectif consiste à analyser les interprétations et les compréhensions du chamanisme par cet écrivain, ancré dans ce phénomène culturel et religieux.

\section{The Literary Representation of Korean Shamanism by Kim Dong-ri (1913-1995)}

The shamanism exerted a major influence on Kim Dong-ri (1913-1995), emblematic figure of the Korean literature of the XXe century. This indigenous religion had flourished and reached its peak during the kingdom of Silla (57 BC - 935 AD) before the introduction of the Buddhism and the Confucianism. Born in Gyeongju, the ancient 
capital of Silla, Kim Dong-ri tries to find in the shamanism the soul of his nation and does not cease sublimating it in his work.

This study aims to answer the following questions: How the shamanism is represented in the literature of Kim? By what means does he develop this religious fact? What is, finally, the relationship between the shamanism and what he calls the "third humanism" or "Korean humanism"? The objective consists in analyzing interpretations and comprehensions of the shamanism by this writer, firmly rooted in this cultural and religious phenomenon.

\title{
Texte intégral
}

Le chamanisme coréen est une religion populaire fondée sur le panthéisme qui s'est perpétuée tout au long de l'histoire. Bien que marqué par des origines probablement sibériennes, il possède ses caractéristiques propres et occupe une place particulière en Corée. Il y était la seule religion dominante, bien avant le confucianisme, le bouddhisme et le taoïsme, introduits de Chine depuis le IVe siècle après J.-C. En tant que religion la plus ancienne, il demeure le fondement spirituel de la culture coréenne, toujours vivant aujourd'hui parmi le peuple :

\begin{abstract}
Le chamanisme dans lequel l'histoire de la révolution cosmique continue à se répéter est un phénomène religieux primitif qui a bien conservé son caractère originel par rapport aux autres religions. En tant que religion matérialisée par les rites religieux de la classe populaire, il est, me semble-t-il, un phénomène vivant actuel imprégné d'une vie profondément enracinée dans l'esprit de la nation coréenne. Si on l'examine selon la perspective de l'histoire de la religion coréenne, le chamanisme, fait religieux essentiel, est un phénomène propre à la nation coréenne apparu avant même l'introduction des religions étrangères.

우주적 회귀의 역사가 반복되고 있는 무속은 그 어느 종교 보다도 종교로 서의 원질이 잘 보존된 원초적 종교현상인 것이다. 무속은 이렇게 민간층 의 종교의식이 집약된 것으로 한민족의 정신 속에 뿌리 깊게 자리잡고 있 는 생활을 통해 생리화한 현재의 살아 있는 종교 현상이라고 보인다. 그리 하여 이와 같은 한민족의 기층적 종교현상인 무속을 한국의 종교사적 입장 에서 본다면, 외래종교가 들어오기 전부터 한민족이 가지고 있었던 조직적 형태의 종교현상은 무속이라는 귀결점에 이른다. ${ }^{1}$
\end{abstract}

2 Pour les Coréens, le chamanisme n'est pas simple sorcellerie ou magie, mais une religion véritable et estimable qui participe de la joie et la tristesse de leur nation. Il dicte plus ou moins la conception de la vie et de la mort ainsi que celle du cosmos, et ce depuis le mythe de Dangun relatif à la fondation de la Corée (en 2333 avant J.-C.). ${ }^{2}$ Les religions étrangères adoptées par les Coréens, tels le bouddhisme, le confucianisme et le christianisme, se sont toutes développées dans cet environnement autochtone chamanique avec lequel une confrontation constante était inévitable. ${ }^{3}$ Après avoir connu ses plus beaux jours au royaume de Silla (57 av. J.-C. - 935), il a été supplanté par le bouddhisme, devenu au VIIe siècle religion d'État, et ensuite par le confucianisme qui a exercé une influence prépondérante sur les couches supérieures de la société, au point que les chamans et leurs familles ont été relégués au plus bas de l'échelle dès le Xe siècle, sous la dynastie de Goryeo (918-1392). Durant la dynastie de Joseon (1392-1910), le chamanisme fut condamné, considéré par les élites confucéennes comme une superstition et une magie. ${ }^{4}$ Confronté à la modernisation depuis l'occupation japonaise 
(1910-1945), il est réprimé par le christianisme et dénigré par les esprits rationnels. Au fil des âges, il accepte, pour sa survie, les religions d'origine étrangère sans perdre son identité religieuse. En même temps, son ouverture lui a permis de s'enrichir grâce à l'apport taoïste, confucéen et bouddhique. Bien que maintes fois menacé par l'afflux des religions étrangères, il s'est maintenu jusqu'à aujourd'hui, puisqu'il n'est pas seulement possession exclusive des chamans, mais propriété commune de la nation coréenne.

Il est à noter que l'époque de Silla a été particulièrement propice au chamanisme coréen, alors à son apogée. Cela a laissé des traces ineffaçables dans sa capitale Seorabeol, ancien nom de Gyeongju. ${ }^{5}$ Kim Dong-ri, originaire de cette région, a été profondément influencé par les multiples facettes de ce monde chamanique. Le chamanisme est à la source même de sa création littéraire, élevé au niveau suprême de l'humanisme coréen. Son pays natal lui offre une source d'inspiration essentielle liée au chamanisme, lequel constitue chez lui le fondement idéologique le plus constant et important. Des œuvres telles que le Munyeodo 무녀도 (Portrait d'une chamane, 1936), le Danggogae mudang 당고개무당 (La Chamane du col Dang, 1958), l'Eulhwa 을화 (La Chamane Eul-hwa, 1978) et le Manja donggyeong 만자동경 (Le Miroir de la reine Seon-deok, 1979), sont tout particulièrement consacrées au chamanisme. ${ }^{6}$ Parmi celles-ci, le Munyeodo est son premier récit traitant de la vie d'une chamane. L'auteur montre un attachement très fort à ce roman court, qu'il le remanie trois fois pour aboutir à l'Eulhwa, long roman, étiré, étoffé et remodelé. Les œuvres qui ne décrivent pas directement l'univers des chamans sont elles aussi imprégnées d'une atmosphère similaire.

$4 \quad$ Comment cette religion ancienne coréenne est-elle représentée dans l'œuvre de Kim Dong-ri ? Par quels moyens valorise-t-il ce fait religieux ? Quel est, enfin, le rapport entre le chamanisme et ce qu'il appelle le " troisième humanisme » ou l'« humanisme coréen »? Tout lecteur de cet auteur est inévitablement confronté à ces questions qui surgissent de façon récurrente. Il est clair que notre approche n'est pas celle d'un historien ou d'un anthropologue qui s'intéressent aux faits réels, aux pratiques rituelles du chamanisme et à leur symbolisme. Il s'agit avant tout d'une analyse de leurs interprétations et compréhensions d'un grand écrivain, marqué par ce phénomène culturel et religieux.

\section{L'incarnation des Hwarang de Silla (Corée antique, 57 av. J. -C. - 935)}

Né à Gyeongju en 1913, mort le 17 juin 1995, Kim Dong-ri fut et reste dans toute l'histoire de la littérature moderne et contemporaine l'un des rares écrivains à réfléchir inlassablement à la nature de l'identité et de la tradition coréennes. Il est ainsi considéré comme l' " écrivain le plus coréen qui soit ». Différent de ses contemporains par ses œuvres et par ses activités, il avait, dès le début de sa carrière d'écrivain, une conviction idéologique qu'il essayait sans cesse de transmettre à travers son ouvre littéraire. Cette conviction, qui avait trait à l'affirmation de l'identité nationale coréenne, justifiait son refus de l'occupation japonaise et ses opinions conservatrices.

$6 \quad$ Ses efforts pour approcher l'esprit du peuple coréen autochtone et du destin 
de l'homme au sein du cosmos font de Kim Dong-ri une figure majeure de l'histoire du roman coréen contemporain, car c'est lui qui en représente le courant le plus influent jusqu'à nos jours. Dans sa défense d'une « littérature pure » sous l'occupation japonaise, dans sa quête de l' " ultime finalité de la vie » (구경(究境)적 삶) propre au destin de l'homme après la Libération (1945) et dans son conservatisme, il n'a cessé de développer une esthétique bien particulière de la littérature coréenne, en participant activement aux débats littéraires et en consacrant toute son énergie à la formation des jeunes écrivains. Ses œuvres, qui marient savoureusement coutumes régionales et valeurs universelles, marquent aux yeux des critiques le point de départ du roman coréen.

7 Kim Dong-ri puise son inspiration dans les différentes religions et philosophies, qu'il interprète à sa manière. Aucun autre romancier coréen n'a accordé autant d'importance à un cadre idéologique aussi varié, alliant chamanisme, christianisme, bouddhisme, géomancie, taoïsme et confucianisme, auquel il se réfère à des degrés divers. De tous ces soubassements religieux et idéologiques, c'est le chamanisme qui donne la plus grande inspiration à son écriture laquelle associe la nature, la nation et les dieux. Fondé sur l'esthétique du temple chamaniste ou sur l'esthétique des dieux traditionnels, son univers sublime artistiquement le chamanisme en saisissant la beauté de la disparition face à sa ruine et à son effacement. Dans ses œuvres consacrées aux chamans, il s'intéresse notamment aux rites religieux des Coréens pendant la pratique desquels le chaman joue le rôle d'intermédiaire entre le divin et l'humain. Les chamanes (mudang 무당 en coréen) telles que Mo-hwa (Munyeodo), Eul-hwa (Eulhwa), Danggogae (Danggogae mudang) et Yeon Dal-rae (Maja donggyeong) sont adombrées au cours de la cérémonie de l'initiation par un esprit divin qui va devenir leur maître. Elles sont devenues chamanes à l'appel des dieux. C'est la voie la plus connue pour devenir chaman en Corée, les deux autres étant liées à la transmission héréditaire ou à la quête personnelle. Elles sont pour lui au cœur de l'idée sur la conception littéraire liée à l'âme coréenne. Dans le Munyeodo et l'Eulhwa, Kim Dong-ri pousse plus loin la description de cette image du chaman coréen. Le second, qui est un remaniement du premier, nous livre en détail l'apprentissage d'une chamane à l'appel des dieux, avec les rites qui l'accompagnent. Le noyau de l'intrigue est identique dans les deux romans, mais les noms des personnages principaux changent : Mo-hwa, Uk-i (son fils) et Nang-i (sa fille) dans le premier texte deviennent respectivement Eul-hwa, Yeong-sul et Wol-hui dans le second.

8 La vie d'Ok-seon, avant de devenir chamane et de prendre Eul-hwa pour pseudonyme, a été une suite de malheurs qui correspondent au schéma traditionnel d'une initiation religieuse : son père meurt dans un tripot et sa mère gagne durement sa vie en faisant des travaux subalternes; elle a eu une liaison hors mariage avec un voisin et met au monde à l'âge de seize ans un fils, Yeong-sul. Parce que la famille du père de son enfant n'autorise pas leur mariage en raison de la situation sociale de la jeune fille, celle-ci est obligée de quitter le village où sa réputation est en cause. Elle s’installe finalement avec sa mère et son fils dans le village de son père, où sa mère ouvre une taverne. Ok-seon se marie avec un homme âgé de 52 ans, riche et honnête, dont c'est le deuxième mariage. Très amoureux de sa nouvelle femme, il meurt dès l'âge de 55 ans. Sa mère, qui s'occupe de son fils à la taverne, est morte empoisonnée 
par un poisson-globe. Après la vente de la taverne de sa mère, elle vient habiter avec son fils dans le village de Jatsil (Baekgok).

L'instabilité de sa vie est encore aggravée par la maladie de son fils survenue après son déménagement. Pour épargner la vie de son fils, elle va prier à l'autel où l'on vénère l'esprit tutélaire du village. Là, elle entend une voix lui demandant d'aller chez la chamane du village : Ppak-ji. Grâce aux gut 굿 (rites chamanistes) qui chassent avec grelots et éventails les mauvais esprits, Yeong-sul est entièrement guéri en dix jours. Or, alors que Yeong-sul se rétablit, Ok-seon doit s'aliter. Les symptômes de sa maladie coïncident exactement avec les épreuves conventionnelles du stade pré-chamanique : elle souffre de violents maux de tête, de troubles du sommeil ; elle perd l'appétit, éprouve tourments divers et sensations d'étouffement. Aucun médicament n'a d'effet.

En général, le candidat à la fonction de chaman voit en rêve les âmes des ancêtres, puis celles des chamans décédés qui le préparent à des révélations ultérieures et l'initient en lui infligeant tortures et terreurs. Une divinité chamaniste se manifeste ensuite au candidat à travers des hallucinations visuelles et auditives. ${ }^{7}$ C'est ce qu'expérimente Ok-seon. Dans son sommeil, elle voit d'abord sa mère défunte. Un bon mois après, une vieille femme décharnée aux cheveux blancs la conduit dans son rêve à la montagne, au champ, à la rivière et dans la forêt. À bout de souffle, elle décide d'aller prier de nouveau devant l'autel. Dans la nuit du troisième jours de prière, la vieille femme maigre réapparaît et lui indique le village du mât totémique situé aux environs de la ville de Gyeongju. Sous le mât où Ok-seon creuse la terre, elle trouve un coffre en pierre dans lequel il y a un miroir en bronze, une paire d'anneaux de jade et un grelot. Par ailleurs, le dos du miroir est inscrit du nom de son propriétaire : Seondo seongmo 선도성모 (la déesse de Seondo). Après la découverte de ces objets rituels, elle continue à faire des cauchemars. Elle se rend encore une fois à l'autel où elle entend une voix devenue familière qui lui demande d'aller chez la chamane Ppak-ji. Celle-ci organise publiquement les cérémonies initiatiques d'Ok-seon qui devient chamane devant un public composé essentiellement de femmes du villages. Grâce au parrainage de Ppak-ji, l'esprit de la déesse (femme immortelle) de la montagne Seondo est transmis à l'élue. Ok-seon, devenue maintenant la Mudang Eul-hwa du fait qu'elle a entendu pour la première fois la voix divine à l'autel du village ainsi nommé, poursuit son apprentissage auprès de son maître Ppak-ji. Plus tard, sa puissance chamanique dépasse celle de son initiatrice, tandis que les rituels d'exorcisme organisés par elle sont réputés dans tout le village et au-delà. Ces étapes de la vie de la chamane, explicites dans l'Eulhwa, ne sont pas détaillées dans le Munyeodo. En revanche, les deux fictions traitent d'une manière singulièrement similaire du retour du fils chrétien et des conséquences qui s'ensuivent : le déclenchement du conflit entre la vieille croyance et le christianisme.

11 L'écriture et la publication du Munyeodo 무녀도 (1936) et de l'Eulhwa 을화 (1978) conduisirent Kim Dong-ri à s'intéresser davantage au monde du chamanisme. Il esseya d'en chercher la trace chez les gens de Silla. L'écriture consacrée aux chamans emprunte alors à la mémoire de son pays natal Gyeongju, parsemé de souvenirs du chamanisme de Silla. Les sources historiques, les contes et les légendes contenus dans le Samguk sagi 삼국사기 (Histoire des Trois Royaumes) rédigé par Kim Bu-sik (1075-1151) et dans le 
Samguk yusa 삼국유사 (Mythologie et littérature des Trois Royaumes) d'Il-yeon (1206-1289), moine bouddhiste de la dynastie de Goryeo (918-1392), lui fournirent les matériaux répondant à son attente. ${ }^{8}$ Ses longues recherches sur l'esprit de Silla se concrétisèrent en 1977 avec la parution du Kim Dong-ri yeoksa soseol 김동리 역사소설 (Les Romans historiques de Kim Dong-ri) comprenant seize nouvelles. Celles-ci dépeignent la vie, les émotions, les désirs, la sagesse, les rêves des gens de Silla, ainsi que les lieux de leurs amours et de leur mort. À l'instar de ses autres œuvres, elles sont très marqués par le thème de l'amour et de la destinée tragique. L'écrivain s'inspire du Samguk sagi et du Samguk yusa, dont la lecture lui permet de se familiariser avec la culture de Silla représentée par les Hwarang et leur doctrine (Hwarangdo), qui offre alors une synthèse du chamanisme, du bouddhisme et du taoïsme. ${ }^{9}$

La culture de Silla n'était pas fondée sur le seul bouddhisme, mais sur une version de cette religion développée et implantée dans le cadre chamanique traditionnel. Développée au profit de la créativité, elle unissait chamanisme et bouddhisme grâce à un processus d'assimilation progressive du second par le premier, plus ancien. Elle résultait de l'évolution complexe du chamanisme. S'épanouissait ainsi une nouvelle culture grâce à cette rencontre créative entre deux sources : l'une, bouddhique, d'origine étrangère, et l'autre, chamanique, traditionnelle. C'était là le trait fondamental de la culture de Silla, dont le Hwarangdo fut le modèle.

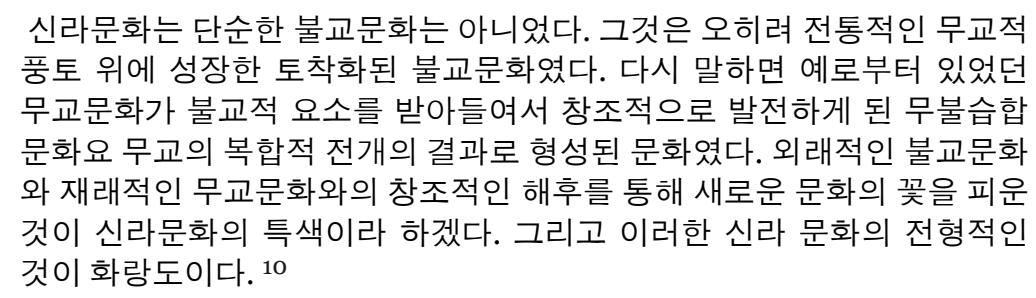

On sait que le royaume de Silla était à l'origine le plus faible et le moins développé des Trois Royaumes. Pourtant, grâce à sa position géographique éloignée de la sphère d'influence chinoise, il adopta facilement les pratiques et les idées du monde non sinisé. Il put plus facilement affirmer sa propre identité et la conserver. Le Hwarang 화랑 花郎 (la fleur de la jeunesse) faisait partie d'une organisation sociale bien structurée, fondée à la fois sur les pratiques nouvelles du bouddhisme et sur le chamanisme. Il constituait l'élite de la jeunesse du royaume de Silla, qui se distinguait par sa conduite et qui excellait dans les arts militaires. C'était une institution religieuse de jeunes gens créée à l'époque du roi Jinheung (540-576). Décidant de nommer des hommes de talent aux postes les plus élevés, le gouvernement de Silla sélectionna deux jeunes filles, Nam-mo et Jun-jeong. Tout en nommant ces dernières «Wonhwa » 원화 (源花), le gouvernement recruta trois milliers de jeunes hommes soumis à leur direction. Peu de temps après, il fut contraint de supprimer cette organisation à cause de la compétition et de la jalousie nées entre les deux jeunes filles : l'une finit par tuer l'autre. Plus tard, le roi pensa néanmoins qu'il fallait avant tout pratiquer l'« art des goûts raffinés » (풍류도 風流道) pour la prospérité du pays. Alors, il ordonna de sélectionner des jeunes hommes issus de la bonne société, se distinguant par leur beauté et leur conduite vertueuse, et de les maquiller. En les nommant Hwarang, le roi attendait du peuple qu'il leur témoigne du respect. Ces Hwarang cultivèrent leur sens moral et voyagèrent entre les monts et les rivières en s'adonnant à la 
musique et à la danse. Le Samguk sagi précise que le Hwarangdo était l'« art des goûts raffinés » fondé sur le chamanisme coréen, enrichi d'éléments issus du taoïsme, du bouddhisme et du confucianisme. ${ }^{11}$

Le chamanisme coréen, dépourvu de doctrine, se trouve facilement rehaussé au contact avec les religions plus théorisées que lui. Les idées en provenance d'autres religions y sont incorporées, de même que des éléments chamaniques s'infiltrent dans le taoïsme et le bouddhisme. Le fait que l'on qualifiait parfois un Hwarang d'homme immortel (선인) ou d'immortel de la nation (국선) témoigne de l'étroite relation entre le Hwarangdo et le taoïsme. ${ }^{12}$ Le célèbre maître bouddhiste Won-gwang (?-630) définit pour les Hwarang les normes de leur conduite, énoncées dans les Sesok ogye (Les cinq commandements pour les séculiers), à savoir la loyauté, la piété filiale, la fidélité, le courage et la bonté, valeurs rejoignant les vertus cardinales confucéennes : servir avec loyauté son souverain (사군이충), servir ses parents avec piété filiale (사친이 효), entretenir fidèlement les liens d'amitié (교우이신), ne pas fuir la guerre (임 전무퇴), ne pas tuer aveuglement les êtres vivants (살생유택).

La fusion du chamanisme et du bouddhisme, caractéristique de la culture de Silla, est chère à Kim Dong-ri, particulièrement attiré par les Hwarang, à la fois chevaliers et chamans, qui jouèrent un rôle de premier plan lors de la guerre de l'unification des Trois Royaumes. Ces héros, en qui se découvre l'origine du chaman coréen, inspirent la plupart des personnages de ses romans historiques. D'ordinaire, ils perfectionnent leur sens moral et se plaisent à écrire de la poésie, de même qu'ils voyagent d'une montagne célèbre à l'autre et pratiquent exercices physiques et intellectuels. En état d'urgence nationale, ils se dévouent entièrement à leur patrie en se rendant dans la montagne pour prier et en allant au front. Kim Yu-sin, Juk Ji, etc, qui se distinguèrent par leurs exploits lors de l'unification de Silla, étaient tous des Hwarang. ${ }^{13}$

Un Hwarang remplit les fonctions suivantes : celle de voyageur se distrayant avec le chant et la danse, celle de chaman qui communique avec les esprits, celle de militaire et celle d'instructeur formant des disciples. ${ }^{14}$ Le chant, la danse et la communication avec les esprits y expriment clairement sa dimension chamaniste. On sait qu'en coréen, mudang 무당 est le terme réservé à la femme chaman, alors que baksu 박수 ou hwarang 화랑 désignent un chaman masculin. Cet usage linguistique illustre encore une fois la prééminence du chamanisme chez les Hwarang de Silla. Leur maquillage semblable à celui de Wonhwa (nom originel du Hwarang), Nam-mo et Jun-jeong, hérite de la tradition chamanique coréenne, car aujourd'hui même, les baksu, minoritaires par rapports à leurs consœurs, se vêtent et se maquillent comme celles-ci selon la coutume transmise par les Hwarang de Silla. ${ }^{15}$

La formation conduisant l'obtention du titre du Hwarang se faisait selon un processus similaire à celle du chaman. Par exemple, Kim Yu-sin (595-673), grand général à l'époque de l'unification de Silla, dut, pour devenir Hwarang, s'entraîner à la prière en voyageant seul dans les montagnes et les forêts. ${ }^{16} \mathrm{Il}$ y rencontra l'esprit de la montagne, qui le guida. Ayant obtenu la force de cet esprit concentrée sur son épée et la protection du génie spirituel, il put enfin accéder au titre du Hwarang à l'âge de 15 ans, soit en 609, la $31^{\mathrm{e}}$ année de règne du roi Jin-pyeong. ${ }^{17}$ Les chamans devaient également suivre une initiation d'une durée déterminée avant de pouvoir exercer leur fonction. Comme Kim Yu-sin, les gens de Silla pensaient que les dieux existaient dans la nature. Les 
Hwarang se rendirent, pour recevoir les insignes des dieux, dans une belle montagne, au bord d'une grande rivière ou sur une terre sacrée où ils pensaient les trouver. ${ }^{18}$

Bien que fondée sur le chamanisme, la doctrine des Hwarang (Hwarangdo) n'était pas seulement une sorcellerie improductive et stérile. Les valeurs morales qu'elle véhiculait stimulaient la création culturelle. On la considère comme la fine fleur de la culture coréenne enracinée dans le chamanisme et nourrie de taoïsme, de bouddhisme et de confucianisme. La fleur du chamanisme coréen en germe dans le mythe de Dangun (fondateur mythique de Gojoseon) s'épanouit enfin dans ce Hwarangdo de Silla à travers lequel Kim Dong-ri, passionné de chamanisme coréen, conçoit ce dernier non comme l'antithèse du christianisme, mais comme une conception spécifique de l'âme collective coréenne. ${ }^{19}$ À travers un monde originaire qui s'étend de l'antiquité jusqu'au présent, il incarne l'esprit de Silla dans lequel il voit le fond religieux qui implique le plus fortement l'âme coréenne. Tout comme son frère aîné Kim Jeong-seol (1897-1966) qui préconise l'« esprit du Hwarang», Kim Dong-ri découvre à son tour la puissance spirituelle qui en émane, une découverte qui le conduit non seulement à s'attacher au chamanisme, mais aussi à enrichir son monde intérieur.

Ses romans historiques sont un véritable hymne à la beauté physique des personnages dont la plupart sont les Hwarang, beaux comme les fleurs. Pour lui, aimer les fleurs, c'est justement appréhender le sens esthétique des gens de Silla :

Qu'est-ce qui constitue la beauté de la Nature ? Ce sont les fleurs. Bien que la montagne, l'eau, l'herbe, le ciel et la lune soient eux aussi dotés d'une belle apparence, rien ne peut attirer nos regards plus que les fleurs. Quelles sont les plus belles créatures ? Les jolies femmes, les jeunes garçons et les jeunes filles séduisants.

Alors, les gens de Silla croyaient que l'esprit de la montagne, amoureux de la beauté dont il ne pouvait se séparer et au sein de laquelle il demeurait, adorait les fleurs, les belles femmes, les jeunes garçons et les jeunes filles, et voulait leur tenir compagnie en leur insufflant son génie. $[\ldots]$ Le secret des dieux des gens de Silla résidait dans les fleurs.Pourquoi est-ce j'aime tant les fleurs ? Parce que [l'esprit de] Hwarang, de la Dame Suro ou du chamanisme coule dans mon sang !

자연에서 아름다운 것은 무엇인가? 꽃이다. 물론 산도 물도 수풀도, 그리고 하늘의 달도 다 아름답지만 꽃 같이 자연의 맨 앞에 서서 사람의 눈길을 끄 는 것은 없다. 사람에서 아름다운 것은 무엇인가? 미녀다. 아리따운 소년 소녀다.

따라서 아름다운 것을 사랑하고 아름다운 것을 함께 하고자 하며 아름다운 것 속에 깃들어 있는 신령님은 꽃과 미녀와 소년소녀를 사랑하고, 그들과 함께 하려고 했었며 그들 속에 깃들어 있다고 신라 사람들은 믿었으리라. $[\ldots]$

신라인의 신의 비밀은 바로 꽃에 있었던 것이다. 내가 이렇게 꽃을 좋아하 는 것도 나의 핏줄 속에 화랑이나 수로부인이나 샤머니즘 같은 것이 흐르 고 있기 때문일까. ${ }^{20}$

Les fleurs sont pour lui l'un des symboles de Silla. Elles jouent ici un rôle d'intermédiaire qui le relie aux Hwarang et au monde du chamanisme. C'est une évocation de la source de vie et un rappel des racines. Grâce à elles, Gyeongju, où Kim Dong-ri se situe dans le moment présent, communique avec le passé, celui du royaume de Silla : il recherche ainsi inlassablement un 
dépassement de la temporalité, que la création littéraire rend possible.

Le passage du temps n'altère en rien ses souvenirs et sa conception des gens et des dieux de Silla, qui circulent à l'infini dans son monde originaire. Ils sont omniprésents dans sa conscience, exaltés par la présence des fleurs, par son imagination qui embellit la réalité. Il s'identifie lui-même aux Hwarang et au peuple de Silla adepte du chamanisme, qui n'a jamais cessé de verser son sang. En ce sens, on peut dire que la religion des chamans n'est pas chez lui un monde acquis, mais plutôt inné. Ainsi s'établit une convergence, une connivence entre son identité et celle des gens de Silla. Son écriture consacrée au chamanisme trouve sa motivation dans la représentation de ses racines au sein du monde de Gyeongju, où les souvenirs de Silla et des Hwarang resurgissent constamment.

21 Ce n'est donc pas par hasard qu'il intitule sa première nouvelle le Hwarang-ui huye 화랑의 후예 (Le Descendant des Hwarang, 1935). Son histoire ne vise pas à glorifier les Hwarang, mais, en choisissant un tel titre, il cherche à rappeler que l'identité des Coréens est marquée par l'esprit du Hwarang, à une époque où toute la Corée sombre dans un sentiment de perte et de néant sous l'occupation japonaise. Parmi ses romans historiques publiés en 1977, les nouvelles Wonhwa 원화 (Deux femmes Hwarang) et Seoktalhae 석탈 해 (Le Roi Seok Tal-hae) décrivent le culte que vouent les Hwarang à leur roi : Hyeok Geo-se, fondateur de Silla. Le Mireukrang 미륵랑 (Le Hwarang Mi-reuk) et le Howonsagi 호원사기 (Récit du temple Howon) remettent en cause l'union du chamanisme et du bouddhisme. De même que Gu-ji et Sae-dal dans le Mireukrang quittent leurs familles pour devenir l'un, moine, et l'autre, nonne, en se promettant de se revoir dans l'autre monde pour réaliser leur amour impossible ici-bas, Kim Hyeon dans le Howonsagi, incapable d'oublier Ho-im, se fait moine après avoir bâti un temple qui porte le nom de Howon, d'où le titre du récit.

22 Outre les seize nouvelles, le Samgukgi 삼국기 (Récit de Trois Royaumes), long roman historique que Kim Dong-ri publia régulièrement à partir du $1^{\mathrm{er}}$ janvier 1972 dans le Seoul sinmun 서울신문 (Le Journal de Séoul), est aussi consacré à l'explication de la force cachée du royaume de Silla, vainqueur de deux autres royaumes plus puissants (Goguryeo et Baekje). Il s'attarde longuement sur la chronique de la vie du célèbre Général Kim Yu-sin, évoqué plus haut, et fait de lui un personnage romanesque en lui attribuant une force surnaturelle. Par ce roman dans lequel sont mêlés l'amour fatal et les exploits des Hwarang, il affirme que le Hwarangdo constitue l'esprit de Silla, caractérisé par la fusion entre le chamanisme, unissant l'homme à dieu, et le bouddhisme, doctrine prônant la transmigration des âmes. C'est sur cette fusion qu'il assoit sa propre conception de la religion, où ce bas monde est transcendé par la vie éternelle. Sans exagérer, on peut dire que l'univers du Munyeodo (Portrait de la chamane, 1936), de l'Eulhwa (La Chamane Eul-hwa, 1978) et du Manja donggyeong (Le Miroir de la reine Seon-deok, 1979) résulte aussi de l'intérêt qu'il porte à Silla, à l'énergie et la vigueur de son esprit. Par ailleurs, ces œuvres nous conduisent directement à la réalité présente, où le destin du chaman est de plus en plus menacé par la modernisation du pays. En mettant en scène la vie des chamanes Mo-hwa, Eul-hwa et Yeon Dal-rae, qui évoquent l'image des Hwarang, Kim Dong-ri souligne la nécessité de ressusciter l'esprit sublime du chamanisme dans la société coréenne contemporaine. 


\section{À la recherche de l'âme coréenne}

Certains commentateurs littéraires considèrent l'écriture de Kim Dong-ri traitant du chamanisme comme une « absence de conscience historique coupée du réel » ou comme une « fuite devant la réalité ». ${ }^{21}$ À ce propos, l'auteur précise dans «Élaboration et fondements du Munyeodo » qu'elle ne consiste pas seulement à faire part d'un souvenir, d'une nostalgie de son pays natal ou de l'émotion suscitée par la vie d'autrefois, mais à exprimer son ambition pour la nation et la littérature coréennes. Son œuvre est avant tout le reflet de l'époque où la Corée était occupée par le Japon. Indigné par la «politique de suppression de la nation coréenne » par l'impérialisme japonais menée à la fin des années 1930, Kim Dong-ri essaie de préserver l'esprit et l'essence de la nation coréenne à travers la littérature en dépeignant l'âme des Coréens sous l'angle du chamanisme. Le Munyeodo 무녀도 (Portrait d'une chamane)s'inscrit dans ce projet. Son cadre temporel est l'année 1907, quand l'Église fut introduite pour la première fois à Gyeongju. L'adoption du christianisme à Seonggeon-dong (lieu de sa naissance) nommé le « village du chaman » n'était guère aisée. On peut imaginer les conflits entre les indigènes et les chrétiens. L'auteur vit de ses propres yeux comment le chamanisme s'était affaibli face à la culture chrétienne pénétrant profondément la vie des Coréens avec la fondation de l'église à Gyeongju. Dès lors, il s'aperçut que le chamanisme transmis depuis les temps anciens passait de plus en plus pour une superstition après l'introduction du christianisme, très fermé à l'égard des autres religions. La création du Munyeodo résulte de cette prise de conscience. C'est cette évolution regrettable qu'il décrit dans cette nouvelle pour manifester en littérature la crainte de voir disparaître le chamanisme à une époque de changement et de confrontation avec les étrangers. Dans cette œuvre qui fait sentir la tristesse de la famille de Mo-hwa, il montre comment une chamane renommée autrefois est dévastée par la montée du christianisme.

Kim Dong-ri est convaincu que la spécificité de la nation coréenne réside dans le chamanisme. À partir de la composition du Munyeodo, il mène des recherches approfondies sur l'univers chamanique avec la certitude que ce dernier peut être considéré comme un archétype de l'âme coréenne. Le chamanisme est pour lui à la source de la vie des Coréens, bien davantage qu'une simple superstition. Il s'en explique ainsi :

En premier lieu, je voulus écrire quelque chose ayant trait à la nation. Étant donné que le régime du gouverneur général était une période d'obscurité supprimant tout ce qui relevait de l'esprit ou du caractère nationaux, je pensais que, malgré une situation où on ne pouvait réellement s'y opposer, je devais les sauver et les défendre au moins par le biais de la littérature. Je réfléchis à la question suivante : quels sont les aspects les plus essentiels et fondamentaux de l'esprit et de l'âme nationaux ? En guise de réponse, je ne songeai pas immédiatement au chamanisme. En découvrant le christianisme en Occident, le confucianisme et le bouddhisme en Orient, tout en considérant la conception de la vie et les valeurs de chaque époque dans les diverses nations, je pensai au cas de la Corée : la question était de savoir ce qui constituait la religion originelle de mon pays avant l'introduction du bouddhisme ou du confucianisme. Je pensai que le chamanisme correspondait à cette étape, à supposer alors que le chamanisme d'aujourd'hui puisse en être considéré comme la religion la plus primitive, il m'apparut que les valeurs spirituelles propres à ma nation 
étaient ancrées dans le chamanisme, et que l'on pouvait le retrouver, le protéger et le faire revivre à notre époque en révélant l'âme de la nation au moyen de l'art (la littérature).

첫째, 민족적인 것을 쓰고자 했다. 당시는 민족정신이라든가 민족적 개성 에 해당되는 모든 것이 말살되어가는 일제 총독 치하의 암흑기였기 때문에 현실적으로 이에 맞설 수 없는 실정이라면 문학을 통해서나마 이를 구하고 지켜야 한다고 생각했던 것이다. 여기서 가장 근본적이며 핵심적인 민족의 얼이요 넋이 되는 것은 무엇일까 하는 문제를 생각하게 되었다. 그렇다고 그 해답으로써 당장 무속을 생각해낸 것은 아니다. 나는 먼저 모든 민족에 있어서 각각 그 시대의 생의 이념이 되고 그 가치의 기준이 되는 것을 흩어 볼 때 서양에서는 기독교 동양에서는 유교 불교 따위란 것을 발견하고, 우 리 한국의 경우를 생각해 보았다. 즉 우리 민족에 있어서 불교나 유교가 들 어오기 이전, 이에 해당하는 민족고유의 종교적 기능을 담당한 것은 무엇 일까 하는 문제였다. 내가 샤머니즘에 생각이 미치게 된 것은 이러한 과정 을 거쳐서였고, 따라서 오늘날의 무속이란 것이 우리 민족에 있어서는 가 장 원초적인 종교적 기능이라고 볼 때 그 가운데는 우리 민족고유의 정신 적인 가치의 핵심이 되는 그 무엇이 내재하여 있을 것이라고 생각했다. 이 것을 찾고 지키고 나아가서는 현대 속에 되살리는 길은 곧 민족의 고유한 혼을 예술(문학)로서 구현시키는 일이라고 믿게 되었다. ${ }^{22}$

Le sentiment de désespoir qui l'étreint lui donne l'occasion de réfléchir à l'âme coréenne enracinée dans le monde des vieilles coutumes et croyances. Contrairement aux critiques des commentateurs, son univers chamanique est conçu avec une pleine conscience de l'histoire et de la réalité. Soucieux de la survie de son pays confronté à des épreuves sans précédent, il décide de transmettre à jamais l'essence de l'esprit national à travers ses œuvres littéraires.

Tout à son amour pour la nation, Kim songe également à l'avenir de la littérature coréenne en décrivant l'univers des chamans :

En deuxième lieu, je voulus relever le défi des problèmes auxquels le monde se heurtait. [...] Parmi les problèmes de la fin du siècle touchant à un sujet sans doute trop vaste et imposant, les plus importants étaient pour moi celui de Dieu et de l'homme, celui de la nature et de la surnature et celui de la science et du mystère.

Tant que ces problèmes concernant « Dieu et l'homme », « la nature et la surnature ", " la science et le mystère », etc., étaient complètement influencés par la conception de Dieu, de la nature, de la science et du mystère élaborée par les Européens sous la bannière du christianisme, je pensais que je ne pourrais pas obtenir de réponse extraordinaire, même si je me lançais dans une nouvelle analyse des mêmes objets et du même cadre. En étudiant la nature de notre chamanisme impérissable, je voulus ouvrir une voie littéraire importante qui puisse offrir une réponse à ces problèmes universels à travers un autre regard sur le divin, la vie et la nature grâce au chamanisme.

둘째, 세계적인 과제에 도전코자 했다. [...] 세기말의 과제라면 대단히 광범 하고 거창한 내용을 가리키게 되겠지만 그 가운데서도 가장 핵심적인 문제 는 신과 인간의 문제요, 자연과 초자연의 문제로 과학과 신비의 문제라고 나는 생각했다.

이러한 <신과 인간>, <자연과 초자연>, <과학과 신비>등등의 문제는 어 디까지나 기독교를 전제한 유럽 사람들의 신관, 인간관, 자연관, 과학관, 신 비관 등등에서 빚어진 만큼 같은 테두리 안에서 같은 대상을 두고는 아무 리 새로운 분석을 시도해 봐야 별로 신통한 대답이 나올 수 없다고 보았다. 여기서 나는 우리의 무속의 영원을 파헤쳐 그 속에 내재되어 있는 신관, 인 생관, 자연관 등으로 이 세계적인 과제에 대처할 수 있는 문학적 혈로를 열 어 보고자 했다. 23 
À travers ces conceptions nouvelles empruntées au chamanisme, il essaie de résoudre les problèmes universels non résolus relatifs aux dieux et aux hommes, à la nature et à la surnature, à la science et au mystère, qui lui paraissent difficiles à expliquer selon les critères de la pensée chrétienne.

Dans la postface du roman Eulhwa 을화, le dernier de Kim Dong-ri, où il traite encore d'une histoire de chamane, il éclaircit plus concrètement l'univers littéraire auquel il aspire en gardant son idée initiale :

Si je veux expliquer sans détour les raisons pour lesquelles j'ai introduit le chamanisme dans mon univers littéraire, je les résumerai par les deux points suivants.

En premier lieu, puisque le chamanisme est la religion primitive des peuples coréens, je pensai que le concept propre à la nation coréenne relatif à dieu, à la vie éternelle, à la vie ici-bas, etc, y auraient leur place. En réaction à la politique du gouverneur général de l'époque, je m'efforçai de perpétuer à jamais l'âme coréenne au moins dans mes œuvres littéraires.

En deuxième lieu, je choisis également le chamanisme pour la nature particulière des dieux que l'on y trouve, puisque le renouveau spirituel ne me semblait possible que grâce à de nouvelles figures divines, alors qu'il m'apparaissait que toutes les religions conventionnelles, celles qui concernent les dieux chinois traditionnels, ne représentaient déjà plus à la fin du XIX ${ }^{\mathrm{e}}$ siècle une source spirituelle de création majeure. Ce que je m'efforçais de montrer du heurt entre chamanisme et christianisme dans le Munyeodo n'était pas un conflit entre cultures orientale et occidentale, ni une opposition entre esprits ancien et nouveau, comme on aurait pu le croire, mais c'était pour moi le moyen d'essayer, au moins à travers l'expression littéraire, de faire naître un nouveau dieu qui proposerait un avenir au monde. [...] À travers l'Eulhwa, je voulus figurer dans la littérature cet univers chamaniste que j'avais simplement évoqué jusque là dans l'atmosphère du Munyeodo, et aussi proposer à la littérature coréenne et mondiale les nouveaux problèmes inhérents à notre monde et à l'au-delà selon le chamanisme.

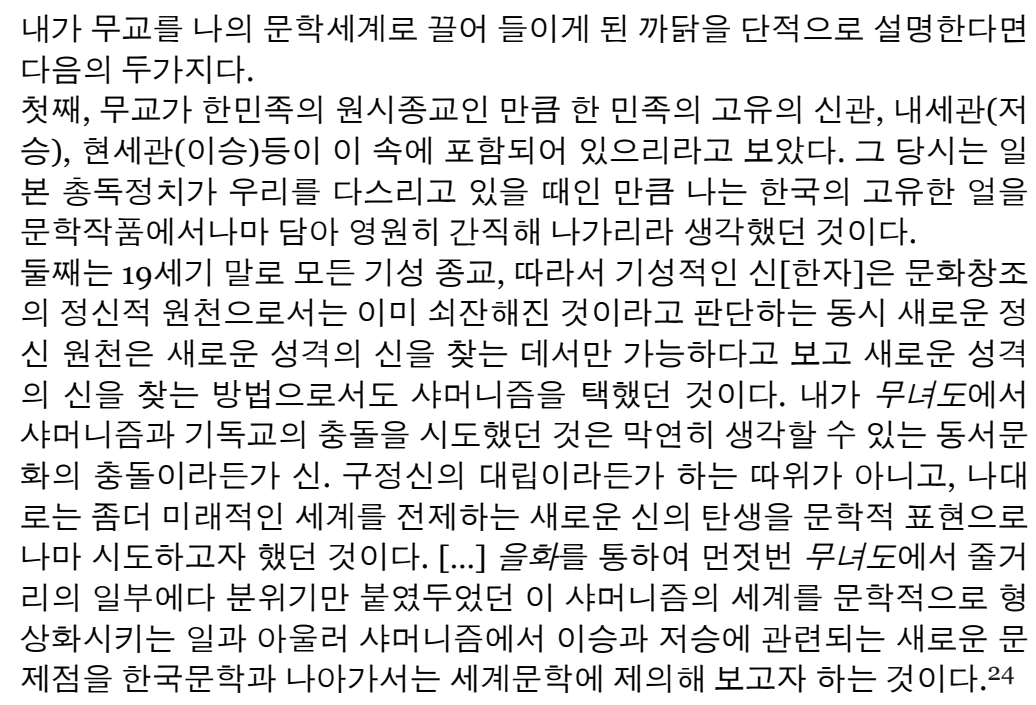

Le chamanisme s'associe dans son univers à la volonté de se libérer de l'impérialisme japonais et d'insister sur les valeurs spirituelles de l'Orient face à celles de l'Occident.

Persuadé que les éléments culturels les plus coréens sont aussi les plus universels, il tente, à travers le Munyeodo et l'Eulhwa, de présenter les dieux orientaux, notamment ceux du chamanisme coréen. Pour lui, c'est un thème 
fort, avec lequel la littérature coréenne peut se mesurer à la littérature mondiale. Considérant le chamanisme comme une conception du monde et de la religion inhérente à la nation coréenne, il emploie toute son énergie à décrire le monde des chamans. Tout en prêtant attention à la nature tragique du chamanisme coréen, il lui accorde une place majeure dans ses œuvres romanesques.

\section{Pour un nouvel humanisme : le « troisième humanisme »}

31 Le chamanisme devient la matière spécifique de ses romans et l'élément déterminant de sa conception de l'humanisme coréen. Dans son Sunsu munhag-ui jinui 순수문학의 진의 (Le Vrai sens de la littérature pure, 1949), il évoque les limites de l'humanisme de l'Occident moderne et la nécessité historique de le renouveler. Il avance la notion de « troisième humanisme » ou de «néo-humanisme » qui est pour lui un dépassement des deux premiers, occidentaux, celui de la Grèce ancienne et celui de la Renaissance. Il les examine l'un après l'autre pour donner du sens à sa pensée et légitimer son nouvel humanisme, fondé sur une figure à la fois divine et humaine, à laquelle il recourt. ${ }^{25}$

32 Selon notre auteur, l'humanisme de l'Antiquité (la période du premier humanisme) établit les valeurs de l'humanité face au sophisme et aux commandements mythiques et superstitieux. La Renaissance (la période du deuxième humanisme) est un mouvement où fleurissent de nouveau les arts et la littérature au temps où le souffle de la modernité commence à se faire sentir et donne l'occasion d'exalter la dignité de l'individu et de vivre une nouvelle expérience qui n'est plus aussi soumise à l'Église qui, au Moyen-Âge, était omniprésente. Les humanistes de l'époque, grâce à la redécouverte de la Grèce, prennent conscience que l'homme est un être de raison. Ce point de vue modifie radicalement la société occidentale sous tous ses aspects. Il exerce une grande influence sur le développement des sciences naturelles. Mais, aux yeux de Kim Dong-ri, l'évolution de la civilisation reposant sur l'essor des sciences naturelles a entraîné l'homme vers le scepticisme depuis la seconde moitié du XIXe siècle. La civilisation contemporaine fondée sur les techniques scientifiques réduit la créativité artistique et menace la faculté de penser et de juger, qui va au-delà du désir de confort de l'homme ; elle introduit en outre une immoralité qui néglige les valeurs et l'éthique enracinées dans la religion, l'art et la philosophie. La mythologie antique et le dieu du Moyen-Âge laissent peu à peu place au scientisme. Un matérialisme triomphant se répand dans la société occidentale et ailleurs. La scientificité n'offre pas à l'homme le véritable bonheur. L'homme moderne est en proie à un sentiment de crise et d'inquiétude et perd le sens de l' "ultime finalité de l'existence ».

Conservateur traditionnel, Kim Dong-ri critique la civilisation moderne occidentale et cherche dans l'esprit oriental un moyen de surmonter la crise du modernisme soumis au matérialisme et au scientisme. ${ }^{26}$ Il considère que la perte du sens de l'humain propre à sa nation est parallèle à celle du monde, qui a aussi connu une période de dévastation des valeurs humaines sans précédent. Seul le chamanisme auquel il s'attache depuis le début de sa carrière est 
capable de redresser l'âme nationale coréenne affaiblie sous l'occupation japonaise. La littérature coréenne ne doit pas donc s'appuyer seulement sur le modèle occidental. Au lieu de professer une idéologie, comme le font la plupart de ses confrères, il l'oriente vers la définition d'une nouvelle divinité, ce qui veut dire que son humanisme revêt un caractère religieux inspiré du chamanisme. C'est dans l'humanisme coréen qu'il cherche à découvrir cette nouvelle divinité, caractérisée par la « fusion entre le ciel et l'homme (천인합 일) 》 :

[...] Devant le constat selon lequel l'humanisme du temps passé s'est essoufflé, il est nécessaire de faire appel à un nouvel humanisme. Quelle en est la nouveauté ? Puisque l'antidivinité constitue la caractéristique essentielle de l'humanisme moderne, il faudrait en inventer les autres qualités.

Qu'en est-il de l'humanisme coréen ? On peut voir qu'il était dès l'origine né d'une collaboration entre le divin et l'homme. Le fait que le dieu du ciel, Hwanung, se soit transformé en humain, prouve que la présence divine est latente en chaque être humain. Son union avec Ungnyeo, ourse transformée en femme, témoigne, quant à elle, du mariage de la divinité et de la nature. Le fondateur de la nation coréenne, Dangun, issu de cette union, était donc le fruit d'un coproduction entre divin et nature en même temps qu'entre divin et humain.

Ainsi, l'humanisme coréen était dépourvu du caractère d' " antidivinité » propre à l'humanisme occidental, mais marqué par l'esprit divin, à savoir que l'homme [coréen] incorpore dès son origine sa dimension divine en lui. C'est que le divin et l'humain provenaient de la nature, leur source commune. [...]

Par conséquent, l'homme et Dieu ne sont pas dans une relation antagoniste, mais dans un rapport aussi étroit que celui unissant un frère et une sœur. C'est la raison pour laquelle l'homme possède une dimension divine. C'est un nouvel humanisme fondé sur une conception de la nature humaine très différente de celle de l'humanisme moderne occidental.

[...] 과거의 인간주의는 갈 데까지 다 갔다는 결론이 나와 있는 셈이니 당연히 새로 운 성격의 인간주의가 오지 않으면 안 될 형편인 것이다. 여기서 새로운 성격이란 무 엇인가. 근대 인간주의의 기본 성격이 ‘반신적’이었기 때문에 성격에 대한 새로움이 오지 않으면 안 되는 것이다.

그런데 한국 인간주의는 어떤가. 처음부터 그것은 신과 인간의 합작이었다고 볼 수 있다. 천신 환웅이 인간으로 화했기 때문에 인간의 내부에는 신이 잠재되어 있음을 말하는 것이다. 곰이 사람으로 된 웅녀와 결혼했다는 것은 신과 자연의 합작이기도 한 것이다. 따라서 거기서 태어난 국조 단군은 신과 인간의 합작인 동시 신과 자연의 합작이기도 했던 것이다.

따라서 한국 인간주의의 성격은 서양의 그것과 같은 “반신적’이 아니고 ‘여신적’이랄 까 신을 내포한 인간 그것이었던 것이다. 그것은 신과 인간이 다 같이 자연을 그 모 체로 삼고 출발했기 때문인 것이다. [...]

따라서 사람과 신은 대립적인 관계에 있는 것이 아니고 동기와 같은 관계인 것이다. 사람이 신을 내포하게 된 연유인 것이다. 이것은 서양의 근대 인간주의와 대조적인 인간관의 새로운 인간주의인 것이다. ${ }^{27}$ transmis de génération en génération à travers le mythe de la fondation de la Corée (le mythe de Dangun, 2333 av. J.-C.), le bouddhisme de Won-hyo (617-686) à l'époque de Silla, et le Cheondogyo (la religion de la Voie du Ciel) de Choe Je-u (1824-1864) et la pensée de Choe Hae-wol (1827-1898) :

La notion de l'esprit du chamanisme est identique à celle de l'esprit du ciel et de la terre. Il s'agit de la pure énergie du ciel, de la terre et des dix 
mille êtres. Dans le Cheondogyo, le Ciel, source de la Voie, est considéré comme l'esprit de l'Univers. Dans ce cas, Choen veut dire à la fois le Ciel, le Seigneur du ciel et l'esprit-dieu. Le ciel, le dieu, l'esprit signifient donc l'énergie de la Nature. Pour les chefs religieux tels que Choe Je-u, Choe Hae-wol et Sin Sa-nae qui s'appuyaient sur le chamanisme de la Corée, le Ciel revient au même que l'Esprit sur un plan sémantique, même si tous l'exprimaient par le caractère chinois tian 天. L'esprit du chamanisme signifie donc l'énergie de la Nature.

샤머니즘의 신령님은 천지신명(天地神明)과 같은 개념이다. 천지만물의 정기(精氣)란 뜻이다. 천도교에서는 ‘도(道)’의 근원인 ‘천(天)’을 가리켜 '만물의 정기’라 한다. 이 경우 ‘천’은 하늘이자 곧 하느님이요, 또한 신(신 명, 神明)이란 뜻이다. 그러니까 천, 신, 신령님은 자연의 정기란 뜻이다. 교 조(教祖) 최제우나 최해월 신사내 다같이 한국 고유의 무교(뜨教)에 바탕 을 두고 있었기 때문에 이분들이 한문 글자로는 천(天)이라 표현했지만 내 용에 있어서는 신령님이나 같은 뜻으로 통해 있다. 그러므로 샤머니즘의 신령님은 곧 자연의 정기란 뜻이다. ${ }^{28}$

Le chamanisme coréen que Kim Dong-ri sublime a évolué en Corée en forme de l'environnement culturel. La danse et les chants, fortement pratiqués dans les cérémonies antiques d'offrandes au Ciel (제천의례), permettent dans le cadre chamanique une " fusion de dieu et de l'homme ». Le Hwarangdo (La doctrine des Hwarang) et le Donghak (mouvement populaire conservateur opposé à toute influence venue d'Occident, en particulier l'influence chrétienne) en sont des exemples probants. Les Hwarang de Silla, à la fois chamans et chevaliers, se consacrent à la protection de la patrie et communiquent avec les dieux à travers le rituel de l'offrande par les chants et les danses qu'ils leurs dédient dans les montagnes célèbres et les grands fleuves. Le Donghak, créé au XIXe siècle par Choe Su-un (Hae-wol), cherche également à sublimer le chamanisme. Cette nouvelle pensée religieuse recueille les religions existant en Corée pour surmonter la crise nationale face aux puissances étrangères. Ce mouvement qui consiste à sauver le pays avec l'aide du Seigneur du ciel est un phénomène religieux adapté de la vieille croyance chamanique liée à la " fusion entre le divin et l'humain ». ${ }^{29}$ Choe Su-un, son leader qui connaîtra une fin tragique, souligne que l'homme fait partie de l'énergie cosmique.

L'esprit du chamanisme coréen traduit ainsi l'harmonie de la "relation ciel-terre-homme (천지인조화) », qui se matérialise à travers les rituels de chants et de danse. Le rêve chamanique d'un monde fondu en une seule instance transcende la division et le dualisme. En examinant l'humanisme coréen originel depuis le mythe de la fondation de la Corée, Kim Dong-ri en vient enfin à identifier la " doctrine de correspondance entre le ciel et les hommes (천인일여설) 》 de Cheo Je-u, qui se réclame de la tradition du chamanisme. L'humanisme coréen est pour lui la clef au problème le plus aigu du monde d'aujourd'hui, même s'il n'est pas systématiquement théorisé ni connu dans le reste du monde. ${ }^{30}$

En vue d'établir une nouvelle conception du monde qui devrait dépasser les théories existantes du XXe siècle, Kim Dong-ri propose l'idée d'un nouveau dieu tel qu'il existait dans le chamanisme coréen, un dieu situé dans la Nature en même temps qu'en l'homme. Les différents noms sont donnés à sa théorie : " troisième humanisme ", " troisième conception du monde " ou "néo-humanisme ». Pour lui, le Dieu occidental ne peut défendre l'humanité en raison de sa dimension absolue et de sa position trop élevée. Il faut donc un dieu qui ne soit ni absolu ni éloigné de l'homme. L'auteur voit dans le monde 
chamanique une conception divine qui possède ces caractéristiques et qui diffère du Dieu occidental. Le dieu dont il est question est un « être doté de l'esprit du dieu ", c'est-à-dire un être humain qui peut librement passer du monde céleste à l'univers du souterrain et à la terre du chaman. ${ }^{31}$ Il apparaît ainsi plus naturel et plus accessible que le Dieu absolu des Occidentaux. Ce dieu qui, pour Kim Dong-ri, peut guider à nouveau le monde à venir est soumis aux règles de la Nature. En épousant le rythme de la Nature, l'homme peut faire l'expérience de la divinité qui lui est inhérente. Quand le rythme de la vie de l'homme s'accorde à celui de l'Univers (la Source), la civilisation et l'art se font jour. L'homme rythmique, c'est-à-dire l'homme qui suit le rythme de l'Univers, possède l'humain et le divin en lui. C'est l'image idéale de l'homme à laquelle aspire Kim Dong-ri.

$38 \quad$ Son écriture reste fidèle aux principes de ce nouvel humanisme. L'Eulhwa 을 화 (La Chamane Eul-hwa) en est une illustration concrète. La chamane Eul-hwa, être associant l'humain au divin est au cœur de sa conception d'une littérature chantant l'âme coréenne. Dans le Saban-ui sipjaga 사반의 십자가 (La Croix de Schaphan), il montre d'abord la distance impossible à réduire entre l'homme (Schaphan), en quête de valeurs terrestres, et le Dieu absolu (Jésus), qui recherche la vie éternelle. On retrouve ce rapport complexe entre l'homme et le divin dans le Deungsinbul 등신불 (Mi-Bouddha, mi-homme), où il décrit une statue de Bouddha qui ressemble à la fois à un homme (par l'apparence) et à un dieu (par l'esprit). Ce roman véhicule l'essentiel de ce qu'il appelle le « troisième humanisme » : récuser le Dieu occidental trop éloigné de la sphère humaine et rejeter les sages orientaux dépourvus de dimension divine, et trop ancrés dans le monde humain. Quant à la chamane Eul-hwa, elle fait l'expérience du divin apportant un secours providentiel. Elle partage la qualité d'être vivant et de divinité. Elle est le fruit de l'union d'homme et de dieu. À travers cette chamane qui joue le rôle d'intermédiaire entre dieu et homme, Kim Dong-ri réalise son idée de " troisième humanisme ».

En effet, Kim Dong-ri est préoccupé par le monde des immortels (선), qui ne peut être expliqué par la science a-religieuse. Ceux qui veulent y accédersont souvent considérés comme des êtres extravagants ou irréalistes à l'affût de la vie qui respecte l'ordre cosmique. Néanmoins, ce sont les héros de ses œuvres de par leurs harmonie avec la nature. Ce monde de l'immortalité est un des traits du chamanisme, car l'image de l'immortel rejoint chez lui celle du chaman :

La Voie de l'immortalité taoïste prit naissance en Corée. L'immortel est l'habitant de la montagne, le montagnard. Seon (l'immortel) peut se prononcer «sen » ou «sae-i » qui signifient mudang (chaman), de même qu'au Gyeongsang-do «san-i » (l'homme de la montagne) est appelé mudang. Les mots «san-i »ou «sen » dérivent du mot «syaman».

Le terme chaman transmis par le monde mongol est un autre mot pour mudang. Parmi ces derniers est nommé " sayan » celui qui sait faire descendre avec facilité des esprits et les recevoir dans son corps ou celui qui est capable de les saisir. [...] Plus tard, transmis en Chine, le terme " sayan " se transforme en chinois en seon (l'immortel) ou sinseon (l’immortel et esprit) [...]. Ce sont des chamans qui se livrent à des pratiques ascétiques.

신선의 선도는 한국에서 발생하였다. 선은 산에 사는 사람, 곧 산인이다. 선 의 음이 '센'이니 '새이'는 무당을 말하고 경상도에서는 ‘산이'가 무당이다. 이 ‘산이’니 ‘센’이니 하는 어원은 근본 ‘샤만’에서 온 것이다. 


\begin{abstract}
몽고계에서 전한 샤만은 곧 무당이란 뜻이다. 무당 중에서 강신이 잘 되는 이를 '사얀’이라고 하며 신 집히는 사람도 '사얀’이라고 한다. [...] 뒤에 이 샤안이 중국으로 들어가서 한자로 선이 되고 또는 신선이라고 하여 [...] 산 에서 수행하는 샤만이 곧 선, 신선이었던 것이다. ${ }^{2}$
\end{abstract}

40 Cette image du chaman et de l'immortel est en concordance avec celle des Hwarang de Silla. L'idéal s'accorde à la réalité grâce à la double identité d'immortel et du chaman. Les Hwarang de Silla apportent donc une ultime réponse au souci d'un humanisme où dieu et homme ne sont plus en contradiction. L'esprit des élites de Silla, modèle idéal de l'homme qui possède le caractère divin, se prolonge par les figures de chamanes que Kim Dong-ri campe dans ses œuvres : Mo-hwa du Munyeodo, Eul-hwa de l'Eulhwa, Dang gogae du Danggogae mudang, Yeon Dal-rae du Manja Donggyeong.

\title{
Conclusion
}

Né dans une contrée riche en légendes et liée au monde chamanique, scolarisé dans des écoles chrétiennes, Kim Dong-ri se lance dans des pérégrinations spirituelles qui oscillent entre Occident et Orient. Aucun autre auteur coréen ne s'est attaché autant que lui au thème du chamanisme dont il fait le point de départ de sa création. Il mêle dans la représentation littéraire qu'il en propose l'histoire, la religion, les pratiques rituelles, les contes ainsi que ses propres souvenirs pour aboutir à une nouvelle forme d'humanisme, une sorte d'universalisme appelé à dépasser les frontières géographiques. Sa quête de l'identité nationale, reposant sur la valorisation des Hwarang de Silla indispensable à ses yeux sous l'occupation japonaise, n'est certes pas dénuée d'une certaine coloration nationaliste, mais elle a pour but de perpétuer cet enracinement coréen dans l'avenir. Dans ce sens, le chamanisme est chez lui à la fois l'origine, le moyen et la fin de son écriture.

La théorie de la littérature de Kim Dong-ri et les œuvres qui la relayent se concentrent sur une conception à la fois immanente et transcendantale du monde. Immanente, parce qu'elle s'intéresse de prime abord à l'homme, à son environnement et à la Nature ; transcendantale, parce qu'elle fait appel aux dieux, aux esprits. L'auteur se tourne ainsi vers l'homme religieux doté d'une divinité immanente. Caractérisée par un aspect non systématique, circulaire et intuitif, sa pensée est une combinaison singulière d'empirisme et d'idéalisme. ${ }^{33}$ Du Munyeodo (1936) à l'Eulhwa (1978), il n'a cessé de s'interroger sur les relations entre la tradition et la modernité, sur les confrontations des religions, sur le destin de l'homme, ce qui constitue la métaphysique de son œuvre, devenue partie inhérente à la littérature coréenne d'aujourd'hui.

\section{Notes}

1 Kim Tae-gon, « Musog-ui jonggyosa-jeok seonggyeok » 무속의 종교사적 성격 (Les caractéristiques du chamanisme à partir de l'histoire des religions), Munhak sasang 문 학사상 (Les Idées littéraires), septembre 1977, p. 347.

2 Le mythe de Dangun inclus dans le Samguk yusa 삼국유사 (Mythologie et littérature des Trois Royaumes), publié par Il-yeon qui était Révérendissime Bogak (moine choisi par le roi comme modèle de la nation) à la fin du XIIIe siècle à l'époque du roi Chungyeol (Goryeo), est une pure création chamanique. 
3 Yu Dong-sik, Hangug-ui jonggyo-wa gidokgyo 한국의 종교와 기독교 (Les Religions coréennes et le christianisme), Séoul, Daehan gidokgyoseohoe, 1965, p. 21-22.

4 Voir pour plus de détail, Cho Hung-yum, « Le chamanisme au début de la dynastie Choson », Cahiers d'Extrême-Asie, nº 6, 1991-1992, p. 1-20.

5 Gyeongju est la ville la plus grande et la plus importante du département de Gyeongbuk, située au sud-est de la péninsule coréenne. Elle a été la capitale du royaume de Silla (57 av. J.-C.-935) pendant mille ans. Dans une grande partie de cette région relativement bien protégée par le gouvernement sont conservés des sites anciens. Lieu historique qui transcende le temps et l'espace, Gyeongju possède une tradition ancestrale marquée par le chamanisme dès l'époque de Gojoseon (Corée ancienne ? -108 av. J.-C.) avant que Silla unifie au VIIe siècle les Trois Royaumes - Baekje (18 av. J.-C.-66o), Goguryeo (37 av. J.-C.-668) et Silla -, dans chacun desquels le bouddhisme a connu un premier développement. L'influence chamanique et bouddhique y est alors prépondérante. Épargnée par le développement urbain et la modernisation, la ville conservait encore, lorsque Kim Dong-ri était enfant, son aspect antique et primitif. Son environnement sacré et inviolable, où les vicissitudes traversées par l'ancienne capitale étaient omniprésentes, exerçaient sur lui une immense fascination.

6 En 1982, lorsque Kim Dong-ri avait 70 ans, l'Eulhwa fut présenté à la sélection du Prix Nobel en littérature grâce à son écriture dotée d'une sensibilité particulière à la tradition coréenne.

7 John et Geneviève T. Park, «Introduction » à La Chamane, traduit de Kim Dong-ri, Eulhwa, Maisonneuve et Larose, 2001.

8 Kim Bu-sik est lettré confucianiste, historien et homme politicien de l'époque de Goryeo. Son Samguk sagi est des écrits les plus anciens sur l'histoire de Corée.

9 Le Hwarang 화랑 désigne les jeunes hommes, fleuron de la jeunesse du royaume de Silla, qui se distinguaient par leur conduite exemplaire et qui excellaient dans les arts militaires. Le Hwarangdo 화랑도 signifie tantôt un groupe de Hwarang, tantôt la doctrine des Hwarang.

$10 \mathrm{Yu}$ Dong-sik, Hanguk mugyo-ui yeoksa-wa gujo 한국 무교의 역사와 구조 (Histoire et structure du chamanisme coréen), Séoul, Presses de l’Université de Yeonse, 1975, p. 82.

11 Kim Bu-sik, «Silla bongi Jinheung wang gisa » 신라본기 진흥왕 기사 (Histoire de l'époque du roi Jinheung de Silla), Hanguk jonggyo-wa gidokgyo (Les Religions coréennes et le christianisme), op.cit., p. 24.

12 Le zen national (국선) : jeune homme choisi comme chef parmi les Hwarang à l'époque de Silla.

13 Yi Chung-geun, Hwarangdo yeongu 화랑도 연구 (Étude de la doctrine des Hwarang), Séoul, 1993, Jongro, p. 11.

14 Yi Cheon-won, «Silla hwarang-ui yeongu » 신라화랑의 연구 (Étude sur les Hwarang de Silla), Joseon sahoe-sa dokbon 조선사회사독본 (Histoire de la société de Joseon), Séoul, Jimmundang, 1990, p. 318.

$15 \mathrm{Yu}$ Dong-sik, Hanguk jonggyo-wa gidokgyo (Les Religions coréennes et le christianisme), op.cit., p. 26.

16 Né à Gyeongju, Kim Yu-sin (595-673) est un descendant de la $12^{\mathrm{e}}$ génération du roi Suro de Gaya et un arrière-petit-fils du roi Guhae de Geumgwan gaya.

17 Kim Bu-sik, «Yeoljeon » 열전 (Les biographies des héros), Samguk sagi 삼국사기 (Histoire des Trois Royaumes), traduit par Kim Jong-gwon, t. 47, pp. 634ff.

18 Kim Dong-ri, Saengga-gi heureun-eun gangmul 생각이 흐르는 강물 (La Rivière de la pensée), Séoul, Gapin chulpansa, 1985, p. 139.

19 Le mythe de Dangun : selon cette belle histoire mythique, la Corée aurait été fondée en 2333 avant J.-C. par Dangun, fils du dieu Hwanung et d'un ours transformé en femme. Le Gojoseon, appelé aussi Dangun Joseon, est le premier royaume de Corée fondé en 2333 avant J.-C. par Dangun.

20 Kim Dong-ri, Kkot-gwa soyeo-wa dal-gwa 꽃과 소녀와 달과 (La Fleur, la fille et la Lune), Séoul, Je 3gihoek, 1994, p. 157-158.

21 Kim U-jong, "Sindang-ui mihak » 신당의 미학 (Esthétique du temple chamanique), Hanguk hyeondae soseol-sa 한국현대소설사 (Histoire du roman coréen contemporain), Séoul, Seonmyeong munhwasa, 1968, p. 277. 
22 Kim Dong-ri, « Musok-gwa na-ui munhak » 무속과 나의 문학 (Le Chamanisme et mon œuvre littéraire), Wolgan munhak 월간문학 (La Littérature), août 1978, p. 151.

23 Ibid., p. 152.

24 Kim Dong-ri, «Eulhwa changjak iyu » 을화 창작이유 (La raison de la création de l'Eulhwa), Munhak sasangsa 문학사상사 (Histoire des idées littéraires), 1978, p. 353-354.

25 Kim Dong-ri, «Sunsu munhag-ui jinui » 순수문학의 진의 (Le Vrai sens de la littérature pure), Seoul sinmun 서울신문 (Le Journal Séoul), le 14 septembre, 1949.

26 Kim Dong-ri, «Chamanisme-gwa bulgyo-wa » 샤머니즘과 불교와 (Le chamanisme et le bouddhisme), Munhak sasang 문학사상 (Les Idées littéraires), 1972, p. 10, 266.

27 Kim Dong-ri, « Hanguk munhak-gwa hanguk inganjuui » 한국문학과 한국인간주의 (La Littérature et l'Humanisme coréens), Kim Dong-ri munhak aelbeom 김동리 문학앨범 (Album littéraire de Kim Dong-ri), Séoul, Unggin chulpansa, 1995, p.166-168.

28 Kim Dong-ri, Kkot-gwa sonyeo-wa dal-gwa 꽃과 소녀와 달과 (Les Fleurs, la fille et la lune), op.cit., p. 161.

29 Yu Dong-sik, Pungryudo-wa hangug-ui jonggyo sasang 풍류도와 한국의 종교사상 (L'Art des goûts raffinés et les pensées religieuses coréennes), Presses de l'Université de Yeonse, 1997, p. 145-151.

30 Kim Dong-ri, Hanguk munhak-gwa hanguk inganjuui 한국문학과 한국인간주의 (La Littérature et l'Humanisme coréens), op.cit., p. 157-168.

31 Kim Dong-ri, Sarang-ui saem-eun got-mada sotgo 사랑의 샘은 곳마다 솟고 (La Source de l'amour jaillit partout), op.cit., p. 165.

32 Kim Jeong-seol, Pungryu jeonsin 풍류정신 (L’Esprit des goûts raffinés), Séoul, Jeongeumsa, 1986, p. 145-146.

33 Yi Dong-ha, Yeoksa-ui jipyeong-gwa cho-yeoksa-ui jipyeong-e daehan haeseol 역사 의 지평과 초역사의 지평에 대한 해설 (Interprétation de l'horizon historique et de l'histoire transcendantale), Séoul, Hakminsa, 1983, p. 218.

\section{Pour citer cet article}

Référence électronique

Min Sook LE , « La représentation du chamanisme coréen chez l'écrivain Kim Dong-ri (1913-1995) », Transtext(e)s Transcultures 跨文本跨文化 [En ligne] , 6 | 2009 , mis en ligne le 07 avril 2011, Consulté le 27 avril 2011. URL : http://transtexts.revues.org /index417.html

\section{Auteur}

\section{Min Sook LE}

Docteur en littérature française et en études coréennes (littérature), LE Min Sook est chargée de cours de coréen à Lyon 3 et membre associé à l'IETT. Elle est l'auteur de Récit et saison chez Colette (L'Harmattan, 2001).

\section{Droits d'auteur}

(C) Tous droits réservés 